OPEN ACCESS

Edited by:

Mohsin Khan,

Temple University, United States

Reviewed by:

Sadia Mohsin,

Lewis Katz School of Medicine at

Temple University, United States

Larisa Emelyanova,

Advocate Aurora Health, United States

*Correspondence:

Lijian Shao

Ishao@ncu.edu.cn

Specialty section:

This article was submitted to

Oxidant Physiology,

a section of the journal

Frontiers in Physiology

Received: 11 August 2020 Accepted: 04 November 2020

Published: 01 December 2020

Citation:

Fan $Y$, Cheng J, Zeng $H$ and Shao L (2020) Senescent Cell

Depletion Through Targeting

BCL-Family Proteins and

Mitochondria.

Front. Physiol. 11:593630.

doi: 10.3389/fphys.2020.593630

\section{Senescent Cell Depletion Through Targeting BCL-Family Proteins and Mitochondria}

\author{
Ying Fan ${ }^{1,2,3}$, Jiaoqi Cheng ${ }^{1,2}$, Huihong Zeng ${ }^{1,2}$ and Lijian Shao ${ }^{1,2,3 *}$ \\ ${ }^{1}$ Department of Occupational Health and Toxicology, Medical College of Nanchang University, Nanchang, China, \\ ${ }^{2}$ Department of Histology and Embryology, Medical College of Nanchang University, Nanchang, China, ${ }^{3}$ Jiangxi Provincial \\ Key Laboratory of Preventive Medicine, Nanchang University, Nanchang, China
}

Senescent cells with replicative arrest can be generated during genotoxic, oxidative, and oncogenic stress. Long-term retention of senescent cells in the body, which is attributed to highly expressed BCL-family proteins, chronically damages tissues mainly through a senescence-associated secretory phenotype (SASP). It has been documented that accumulation of senescent cells contributes to chronic diseases and aging-related diseases. Despite the fact that no unique marker is available to identify senescent cells, increased p16 ${ }^{\text {INK4a }}$ expression has long been used as an in vitro and in vivo marker of senescent cells. We reviewed five existing p16 ${ }^{\text {INK4a }}$ reporter mouse models to detect, isolate, and deplete senescent cells. Senescent cells express high levels of anti-apoptotic and pro-apoptotic genes compared to normal cells. Thus, disrupting the balance between anti-apoptotic and pro-apoptotic gene expression, such as ABT-263 and ABT-737, can activate the apoptotic signaling pathway and remove senescent cells. Mitochondrial abnormalities in senescent cells were also discussed, for example mitochondrial DNA mutation accumulation, dysfunctional mitophagy, and mitochondrial unfolded protein response (mtUPR). The mitochondrial-targeted tamoxifen, MitoTam, can efficiently remove senescent cells due to its inhibition of respiratory complex I and low expression of adenine nucleotide translocase-2 (ANT2) in senescent cells. Therefore, senescent cells can be removed by various strategies, which delays chronic and aging-related diseases and enhances lifespan and healthy conditions in the body.

Keywords: senescence, aging, senolytics, apoptosis, p16 1NK4a

\section{INTRODUCTION}

In the 1960s, Hayflick documented that normal human fibroblasts could not eternally grow, with irreversible cell cycle arrest occurring at a certain point, which is also known as replicative senescence (Hayflick and Moorhead, 1961; Hayflick, 1965). During cell division, telomere length shortens, which contributes to replicative senescence (Olovnikov, 1973; Lange, 2005). It is well accepted that cellular senescence could also be induced by various stressors, such as oncogenic, oxidative, and genotoxic conditions (Hernandez-Segura et al., 2018; Magalhaes and Passos, 2018), which is called premature senescence. Premature senescence is not related to telomere shortening. Replicative and premature senescence have shared features, which include an enlarged and flattened shape, senescence-associated beta-galactosidase (SA- $\beta$-gal) positive staining, and 
increased p16 $6^{\text {INK4a }}$ expression (Hernandez-Segura et al., 2018). Upregulated levels of $\mathrm{p} 16^{\mathrm{INK} 4 \mathrm{a}}$ expression inhibits the formation of catalytically active cyclin $\mathrm{D}$-CDK complexes. It subsequently inactivates cyclin E-CDK2 and cyclin A-CDK2. Inhibition of cyclin E-CDK2 and cyclin A-CDK2 blocks G1 to S-phase transition and results in a G1-phase cell-cycle arrest and cellular senescence (Sherr, 2000; Ortega et al., 2002). Therefore, p16 ${ }^{\text {INK4a }}$, coexisting with increased SA- $\beta$-gal activity, is often used as a robust senescence marker in various mouse and human tissues.

Senescent cells avoid apoptosis and necrosis despite their high levels of metabolism, DNA damage, and senescenceassociated secretory phenotype (SASP) (Dörr et al., 2013; Wiley and Campisi, 2016). Additionally, senescent cells, but not healthy and replicating cells, are resistant to harsh microenvironments, such as nutritionally depleted conditions (Sagiv et al., 2013). These characteristics keep them alive in the body for a long time, which might lead to chronic diseases and age-related dysfunction. It has been shown that an increased number of senescent cells in tissues is correlated to many age-related diseases, such as chronic pulmonary disease, chronic bone marrow injury, osteoarthritis, atherosclerosis, neurodegenerative disorders, chronic renal diseases, and diabetes (Childs et al., 2017; He and Sharpless, 2017; McHugh and Gil, 2018; Barnes et al., 2019; Docherty et al., 2019). Although senescent cells only occupy around $15 \%$ of dysfunctional tissues, the accumulation of senescent cells potentially causes chronic tissue dysfunction, promotes tissue destruction and aging, causes local inflammation, and can even lead to tumorigenesis and metastasis (Herbig et al., 2006). The negative effects associated with senescent cells might be attributed to SASP, indicating that these cells secrete various chemokines, pro-inflammatory cytokines, and extracellular matrix proteases (Coppé et al., 2008, 2010).

The existence of senescent cells has double-edged effects. On the one hand, the short-term appearance of senescent cells has a positive function in suppressing tumorigenesis during early stages (Kang et al., 2011; Baker et al., 2017; Calcinotto et al., 2019), accelerates wound healing (Adams, 2009; Demaria et al., 2014), and maintains tissue integrity during embryonic development (Munoz-Espin et al., 2013; Storer et al., 2013; Davaapil et al., 2017). On the other hand, long-term accumulation of senescent cells is deleterious to tissues through the SASP (Herranz and Gil, 2018; Gorgoulis et al., 2019). Senescent cells are usually removed by the immune system instead of apoptotic and necrotic mechanisms (Burton and Stolzing, 2018; Prata et al., 2018). However, senescent cells will not efficiently be cleared in various tissues if the capacity of the immune system is dysfunctional or if existing senescent cells exceed the clearing capacity of the immune system. Thus, we must develop efficient approaches, such as in vivo depletion of senescent cells, to rejuvenate tissue function.

It is necessary to understand how the existence of senescent cells is regulated and what underpins the molecular mechanisms of these cells in order to discover new strategies that allow selective depletion of senescent cells in vitro and in vivo. The clearance of senescent cells has been shown to delay and reduce various tissue aging phenotypes in premature and natural aged models, such as BubR1 and
Ercc1 knockout progeroid mice (Niedernhofer et al., 2006; Baker et al., 2008, 2011; Childs et al., 2016). Efficient clearance will create good opportunities to assess the functional importance of cellular senescence in different pathological conditions, potentially leading to novel therapeutics. In this review, we discuss five different $\mathrm{p} 16^{\mathrm{INK} 4 \mathrm{a}}$ reporter mice and various known pharmacological strategies to clear senescent cells. We also highlight the importance of targeting mitochondria in selectively depleting senescent cells. Underlying mechanisms of SASP in senescent cells was not discussed here as they have been extensively reviewed by others (Tchkonia et al., 2013; Gorgoulis et al., 2019).

\section{MONITORING AND CLEARING SENESCENT CELLS}

The importance of senescent cells in aging and age-related diseases motivated investigators to find a proper reporter system to monitor and identify senescent cells in vitro and in vivo. First, the essential senescent markers should be identified, as there is currently no common senescent marker in different cells or tissues. Hence, several different methods have to be used to properly define senescent cells. This includes: flattened and enlarged morphology (Munoz-Espin and Serrano, 2014; Simay et al., 2018); SA- $\beta$-gal positive staining (Dimri et al., 1995; Kurz et al., 2000); increased p16 $6^{\mathrm{INK} 4 \mathrm{a}}$ expression (Krishnamurthy et al., 2004; Ressler et al., 2006; Liu et al., 2009); DNA damage (Sedelnikova et al., 2004; d’Adda di Fagagna, 2008; Hewitt et al., 2012); and SASP-related factors (Coppé et al., 2010; Tchkonia et al., 2013; Hernandez-Segura et al., 2018). Thus far, it is well accepted that the induction of $\mathrm{p} 16^{\mathrm{INK} 4 \mathrm{a}}$ expression is a hallmark of cellular senescence in most scenarios. Currently, there are five different $\mathrm{p} 16^{\mathrm{INK} 4 \mathrm{a}}$ reporter mice available, as discussed below (Table 1).

\section{p16-Luc Transgenic Mouse}

In 2009, Dr. Hara's group used a genomic DNA segment containing the entire human INK4a/Arf gene locus to engineer a fusion protein with firefly luciferase (Luc, named p16-Luc) without deleting any genomic DNA sequences at the INK4a/ Arf gene locus, generating transgenic p16-Luc mouse (Yamakoshi et al., 2009). Advantages of this model include: (1) p16-Luc fusion protein was used to specify $\mathrm{p} 16^{\mathrm{INK} 4 \mathrm{a}}$ gene expression instead of $\mathrm{p} 19^{\mathrm{INK} 4 \mathrm{~d}}$ gene expression in their overlapping gene locus; (2) luciferase activity was used to monitor $\mathrm{p} 16^{\mathrm{INK} 4 a}$ expression timely; and (3) $\mathrm{p} 16^{\mathrm{INK4a}}$ family genes $\mathrm{p} 15^{\mathrm{INK} 4 \mathrm{~b}}, \mathrm{p} 18^{\mathrm{INK} 4 c}$, and $\mathrm{p} 19^{\mathrm{INK} 4 \mathrm{~d}}$ did not disrupt $\mathrm{p} 16^{\mathrm{INK} 4 \mathrm{a}}$ gene expression in this reporter. Luciferase activity of mouse embryonic fibroblasts (MEFs) from p16-Luc mice was significantly increased by the induction of cellular senescence. Endogenous mouse p $16^{\mathrm{INK} 4 \mathrm{a}}$ expression was also increased in senescent MEFs. These data imply that the expression of human $\mathrm{p} 16^{\mathrm{NK} 4 \mathrm{a}}$ represents mouse cell senescence. Using the reporter, it confirmed that the increased $\mathrm{p} 16^{\mathrm{INK} 4 \mathrm{a}}$ gene expression could inhibit tumorigenesis and was not derived from cell culture conditions in cultured 


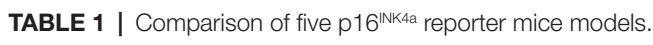

\begin{tabular}{|c|c|c|c|c|}
\hline P16 ${ }^{\text {INK4a }}$ reporter mice & Genetic strategy & Reporter & Features & Refs \\
\hline P16-Luc & Transgenic & Luciferase & Monitoring senescent cells timely & Yamakoshi et al., 2009 \\
\hline P16 & Knockin & Luciferase & $\begin{array}{l}\text { Monitoring senescent cells timely } \\
\text { 1. Isolating and analyzing single senescent cell }\end{array}$ & Burd et al., 2013 \\
\hline P16 ${ }^{\text {tdTom/+ }}$ mice & Knockin & tdTomato & $\begin{array}{l}\text { 2. Fluorescence of tdTom is six times brighter than green } \\
\text { fluorescent protein } \\
\text { 1. Isolating and analyzing single senescent cell }\end{array}$ & Liu et al., 2019 \\
\hline INK-ATTAC mice & Transgenic & GFP, ATTAC & $\begin{array}{l}\text { 2. Clearing senescent cells by AP20187 } \\
\text { 1. Monitoring senescent cells timely. }\end{array}$ & Baker et al., 2011 \\
\hline P16-3MR mice & Transgenic & Luciferase, mRFP, HSV-TK & $\begin{array}{l}\text { 2. Isolating and analyzing single senescent cell } \\
\text { 3. Clearing senescent cells by GCV }\end{array}$ & Demaria et al., 2014 \\
\hline
\end{tabular}

cells (Gil and Peters, 2006; Ohtani et al., 2010). These results resolved the challenge of whether the increased $\mathrm{p} 16^{\text {INK4a }}$ gene expression in tissue culture was a reflection of cellular senescent status and anti-cancer process.

To test the functional ability in vivo, they used naturally aging p16-Luc mice to measure luciferase activity. The data implies that the bioluminescent signaling level was dramatically increased in p16-Luc mice during natural aging. Importantly, they found that oncogenic Ras activation caused epigenetic derepression of $\mathrm{p} 16^{\mathrm{INK} 4 \mathrm{a}}$ expression through reduction of DNA methyl transferase 1 (DNMT1) levels in vivo (Yamakoshi et al., 2009). By using a DMBA/TPA-induced papillomas mouse model, they proved that $\mathrm{p} 16^{\mathrm{INK} 4 \mathrm{a}}$ upregulation could efficiently prevent malignant conversion of benign tumors. This is due to the reduction of histone 3 Lys9 demethylation (H3K9me2) in papillomas, which leads to increasing $\mathrm{p} 16^{\mathrm{INK} 4 \mathrm{a}}$ expression. In the case of p53 mutation, the timeframe of $\mathrm{p} 16^{\mathrm{INK} 4 \mathrm{a}}$ upregulation is much shorter. This indicates that $\mathrm{p} 16^{\mathrm{INK} 4 a}$ expression, as a backup tumor suppressor, is regulated by p53 activity (Yamakoshi et al., 2009). Therefore, cellular senescence is an anti-tumor defense mechanism prompting oncogenic cells into cell cycle arrest.

\section{p16 ${ }^{\text {LUC }}$ Knockin Mouse}

In 2013, Dr. Sharpless's group exploited the highly dynamic induction of $\mathrm{p} 16^{\text {INK4a }}$ observed in response to oncogenic insult and senescence. They used a "knock in" strategy to generate an in vivo reporter system, p16 ${ }^{\mathrm{LUC}}$ (Burd et al., 2013). The firefly luciferase cDNA followed by an SV40 polyadenylation signal was targeted into the translational start site of the endogenous $\mathrm{p} 16^{\mathrm{INK} 4 \mathrm{a}}$ locus. In contrast to previous transgenic reporter systems driven by fragments of the $\mathrm{p} 16^{\mathrm{INK} 4 \mathrm{a}}$ promoter, they preserve the known cis-regulatory elements centromeric to the $\mathrm{p} 16^{\mathrm{INK} 4 \mathrm{a}}$ open reading frame by the "knock in" strategy. The $\mathrm{p} 16^{\mathrm{LUC}}$ allele was utilized to detect senescence and the earliest steps of tumorigenesis in well-defined murine systems.

To examine the correlation between $\mathrm{p} 16^{\mathrm{INK} 4 \mathrm{a}}$ expression and age, $\mathrm{p} 16^{+/ \mathrm{LUC}}$ mice at 16 weeks of age were used to monitor luciferase activity during the mouse natural aging process. The results showed that the luciferase activity was significantly increased during a period of 80 weeks, indicating that $\mathrm{p} 16^{\mathrm{LUC}}$ expression represents a faithful and age-related increase in p $16^{\text {INK4a }}$ gene expression. It is well known that both wound healing and mammary involution models have high $\mathrm{p} 16^{\mathrm{INK} 4 \mathrm{a}}$ gene expression (Gadd et al., 2001; Adams, 2009; Demaria et al., 2014). p16 ${ }^{\mathrm{LUC}}$ reporter in terms of wound healing and mammary involution models showed that increased luciferase activity was easily detected during both processes, while the activity was significantly reduced upon completion of wound healing and mammary involution. Senescent cells secrete plateletderived growth factor AA (PDGF-AA) to induce myofibroblast differentiation, leading to wound closure. Consistently, application of recombinant PDGF-AA onto a wound area promotes its recovery by inducing myofibroblast differentiation (Demaria et al., 2014). These results indicate that cellular senescence has some beneficial roles during tissue repair by secreting SASP factors.

Importantly, the $\mathrm{p} 16^{\mathrm{LUC}}$ targeting strategy with an allele null for $\mathrm{p} 16^{\mathrm{INK4a}}$ does not affect expression of $\mathrm{p} 15^{\mathrm{INK} 4 \mathrm{~b}}$ and $\mathrm{p} 19^{\mathrm{INK} 4 \mathrm{~d}}$. The luciferase activity of $\mathrm{p} 16^{\mathrm{LUC}}$ both in vitro and in vivo robustly reflects endogenous $\mathrm{p} 16^{\mathrm{INK} 4 \mathrm{a}}$ expression. In addition, previous studies have shown that $\mathrm{p} 16^{\mathrm{INK4a}}$ expression was remarkably increased in the early stage but not advanced stage of tumor development (Collado et al., 2007). p16 ${ }^{\text {LUC }}$ reporter will provide an opportunity to detect early-stage tumors, monitor cancer progression, and test anti-cancer drugs. The data further confirms that $\mathrm{p} 16^{\mathrm{INK4a}}$ is a tumor suppressor, limiting cancer progression and cell proliferation. Therefore, $\mathrm{p} 16^{\mathrm{LUC}}$ reporter can be used to dynamically monitor cells undergoing senescent progression under physiological and pathological settings.

\section{p16 ${ }^{\text {tdTom/+ }}$ Knockin Mouse}

As mentioned above, both $\mathrm{p} 16$-Luc and $\mathrm{p} 16^{\mathrm{LUC}}$ reporters have the ability to monitor senescent cells with $\mathrm{p} 16^{\mathrm{INK} 4 \mathrm{a}}$ overexpression. However, both reporters cannot be used to isolate and analyze individual senescent cells based on luciferase activity. To this end, Dr. Sharpless's group generated a murine p16 ${ }^{\text {INK4a }}$ reporter mice by knocking in a tandem-dimer Tomato (tdTom) into exon 1a of the p16 ${ }^{\text {INK4a }}$ locus in 2019 (Liu et al., 2019). They used the reporter line to enumerate, isolate, and characterize the individual $\mathrm{p} 16^{\mathrm{INK} 4 \mathrm{a}}$-expressing $\mathrm{tdTom}^{+}$ cells. The frequencies of $\mathrm{tdTom}^{+}$cells were increased with serial passage in cultured MEFs from $\mathrm{p} 16^{\mathrm{tdTom} /+}$ mice in vitro. They also used a peritoneal inflammation mice model to 
demonstrate that $\mathrm{tdTom}^{+}$macrophages exhibited some features of senescence in vivo, which are reduced proliferation, SA- $\beta$-gal positive staining, and increased mRNA expression of a subset of transcripts encoding factors involved in SASP, such as MMP12 and Cxcl12 (Liu et al., 2019). These results indicate that cells harboring activation of the $\mathrm{p} 16^{\mathrm{INK} 4 \mathrm{a}}$ promoter accumulate with aging and inflammation in vivo, and display characteristics of senescence. Moreover, tdTom fluorescence is six times brighter than green fluorescent protein (GFP) without the predisposition to aggregation and toxicity. Another feature in the $\mathrm{p} 16^{\text {tdTom/+ }}$ knockin reporter is that the expression of tdTom faithfully assesses not only $\mathrm{p} 16^{\mathrm{INK4a}}$ mRNA transcript abundance but also p16 $6^{\mathrm{INK} 4 \mathrm{a}}$ promoter activation. The half-life of tdTom mRNA is about $12 \mathrm{~h}$, whereas that of $\mathrm{p} 16^{\mathrm{INK} 4 \mathrm{a}}$ and p19 ${ }^{\text {INK4d }}$ mRNA is up to $24 \mathrm{~h}$ (Hara et al., 1996; Liu et al., 2019). p16 ${ }^{\text {tdTom/+ }}$ knockin reporter does not reflect those senescent cells with low $16^{\mathrm{INK} 4 \mathrm{a}}$ promoter activity. Thus, the reporter cannot detect all senescent cells in vitro and in vivo.

In comparison to $\mathrm{p} 16-\mathrm{Luc}$ and $\mathrm{p} 16^{\mathrm{LUC}}$ reporter mice, $\mathrm{p} 16^{\mathrm{tdTom} /+}$ reporter allows the detection and isolation of individual senescent cells that express bright tdTom fluorescence. Hence, $\mathrm{p} 16^{\mathrm{tdTom} /+}$ reporter is a perfect tool to perform downstream analysis in senescent cells, such as single cell RNA-Seq, or to find new senescent markers for future studies. p16-Luc mouse model from Dr. Hara's group and p16 ${ }^{\mathrm{LUC}}$ mouse model from Dr. Sharpless's group can be used for timely monitoring of senescent cell appearance in vitro and in vivo. Therefore, $\mathrm{p} 16^{\mathrm{tdTom} /+}$ mouse model provides a good opportunity to detect, isolate, and analyze single senescent cells. However, all three models do not have the ability to clear senescent cells in vivo.

\section{INK-ATTAC Transgenic Mouse}

In 2011, Dr. van Deursen's group developed a transgenic mouse model with features of both monitoring and clearing senescent cells in order to examine the role of cellular senescence in aging and age-related diseases (Baker et al., 2011). They designed a transgenic strategy of constructing FKBP-Caspase8-Flag fusion protein and EGFP under the control of a 2616-bp fragment of the $\mathrm{p} 16^{\text {INK4a }}$ promoter (named INK-ATTAC mice). A synthetic drug AP20187 induces dimerization of a membrane-bound myristoylated FK506-binding protein-caspase 8 (FKBP-Casp8) fusion protein expressed specifically in senescent cells via the control of $\mathrm{p} 16^{\mathrm{INK} 4 \mathrm{a}}$ promoter. BubR $1^{\mathrm{H} / \mathrm{H}}$, INK-ATTAC mice were generated by crossing INK-ATTAC mice with BubR1 mice, which is a progeroid mouse model. $\mathrm{GFP}^{+}$and $\mathrm{GFP}^{-}$cell populations from inguinal adipose tissue in aged $\mathrm{BubR} 1^{\mathrm{H} / \mathrm{H}}$, INK-ATTAC mice were separated to measure $\mathrm{p} 16^{\mathrm{INK} 4 \mathrm{a}}$ expression. Expression of $\mathrm{p} 16^{\mathrm{INK4a}}$ were higher in $\mathrm{GFP}^{+}$cells than $\mathrm{GFP}^{-}$ cells isolated from aged BubR $1^{\mathrm{H} / \mathrm{H}}$, INK-ATTAC mice. In addition, $\mathrm{GFP}^{+}$cells were also detected in primary MEFs with ectopic expression of oncogenic Ras or serial passaging to induce cellular senescence. Taken together, these results indicate that INK-ATTAC is selectively expressed in $\mathrm{p} 16^{\mathrm{INK4a}}$-positive senescent cells.

Elimination of $\mathrm{p} 16^{\mathrm{INK4a+}}$ cells from non-progeroid mice using ATTAC by AP20187 could be used to address how senescent cells influence health and lifespan (Baker et al., 2016). Interestingly, AP20187 treatment delayed cataract formation in both males and females with a C57BL/6 background and reduced glomerulosclerosis independent of sex and genetic background. Depletion of senescent cells is correlated with attenuated age-related blood urea nitrogen increase, which benefits kidney functional recovery (Baker et al., 2016). The negative effect of age-related accumulation of $\mathrm{p} 16^{\mathrm{INK4a+}}$ cells was evidenced by the observation of lifespan extension in AP20187-treated INK-ATTAC mice (Baker et al., 2016).

The healthy condition of transgenic INK-ATTAC mice can be enhanced by selectively killing senescent cells with AP20187. The accumulation of senescent cells in association with several diseases, disabilities, and chronological aging is likely to contribute to the causation and pathophysiology of these problems or their symptoms (Childs et al., 2015, 2017). Together with chronic, sterile inflammation, macromolecular dysfunction, and stem and progenitor cell dysfunction, cellular senescence may contribute to both aging phenotypes and increased susceptibility to a range of chronic diseases (Tchkonia and Kirkland, 2018). The clearance of senescent cells has shown beneficial effects in vivo, such as slowing the development of kyphosis, cataracts, and lipodystrophy in progeroid mice (Baker et al., 2011).

The INK-ATTAC mice were also used to examine the effects of removing senescent cells on bone loss, aged cardiac deterioration, aged pancreatic $\beta$-cells, and other pathological conditions (Farr et al., 2017; Aguayo-Mazzucato et al., 2019; Lewis-McDougall et al., 2019). Elimination of senescent cells with AP20187 treatment benefits the increment of bone mass, activation of resident cardiac progenitor cells, and improvement of pancreatic $\beta$-cell function. These beneficial effects of depleting senescent cells contribute to the decrease of SASP program and expression of senescent markers. Compared to p16-Luc and $\mathrm{p} 16^{\mathrm{LUC}}$ mouse models, the INK-ATTAC mouse model has the notable advantage of clearing senescent $\mathrm{GFP}^{+}$senescent cells. However, the mouse model fails to timely monitor senescent cells through luciferase activity in living animals.

\section{p16-3MR Transgenic Mouse}

In 2014, Dr. Campisi's group developed a reporter mouse with three features: monitoring, isolating, and depleting senescent cells (Demaria et al., 2014). They generated transgenic p16-3MR mice, which contain functional domains of a synthetic Renilla luciferase (LUC), monomeric red fluorescent protein (mRFP), and truncated herpes simplex virus 1 (HSV-1) thymidine kinase (HSV-TK) under the control of $\mathrm{p} 16^{\mathrm{INK} 4 \mathrm{a}}$ promoter (Demaria et al., 2014). LUC allows the detection of $3 \mathrm{MR}$-expressing cells by luminescence both in vitro and in vivo. mRFP allows the isolation of senescent cells from tissues. HSV-TK allows for the killing effects of ganciclovir (GCV), a nucleoside analog that has a high affinity for HSV-TK but a low affinity for the cellular TK. HSV-TK converts GCV into a toxic DNA chain terminator in nondividing senescent cells. GCV fragments mitochondrial DNA, causing death by apoptosis (Laberge et al., 2013).

The $3 \mathrm{MR}$ transgene activity was confirmed using MEFs from p16-3MR mice, which were induced to undergo senescence after irradiation (Demaria et al., 2014). Cellular senescence was verified by SA- $\beta$-gal positive staining and increased expression 
of p16 ${ }^{\mathrm{INK} 4 \mathrm{a}}, \mathrm{p} 21^{\mathrm{Cip} 1}$, and SASP factors. Senescent p16-3MR MEFs, compared to non-senescent cells, have higher levels of luciferase activity with mRFP expression shown via immunostaining. Moreover, GCV selectively removed senescent p16-3MR MEFs without toxicity on non-senescent p16-3MR MEFs. Increased luciferase activity was observed in living animals and different organs such as visceral fat, kidneys, and lungs at 3 months after 7.0 Gy irradiation (Demaria et al., 2014). The expression of p16, mRFP, and SASP factors in irradiated organs was significantly increased. GCV treatment in irradiated p16-3MR mice markedly reduced total body luminescence and senescenceassociated gene expression in fat, kidneys, and lungs. In naturally aged mice model, p16-3MR mice showed a significant increase in luciferase activity starting at 18 months of age. GCV treatment in 20-24-month-old mice efficiently eliminated senescent cells determined by reduced luminescence, SA- $\beta$-gal positive staining, and $\mathrm{p} 16^{\mathrm{INKk4a}}$ expression in visceral fat (Demaria et al., 2014). Thus, these findings show that p16-3MR transgenic mice represent the presence of senescent cells through luminescence assay and allow the removal of senescent cells via GCV treatment.

Currently, the p16-3MR mouse model is largely used to explore the effects of senescence clearance under different pathological settings. Jeon et al. found that senescent cells were present in articular cartilage and synovium in osteoarthritis mouse model (Jeon et al., 2017). Clearance of these senescent cells via GCV treatment significantly increased cartilage development and pain reduction, which attenuates osteoarthritis development (Jeon et al., 2017). In intervertebral degeneration (IDD) model of p16-3MR mice, Patil et al. demonstrated that luciferase activity was high in the intervertebral discs with increasing SASP, which might be attributed to the development of IDD (Patil et al., 2019). Elimination of senescent cells by GCV treatment significantly decreased senescent cells with reducing SASP and disc aggrecan proteolytic degradation, increasing proteoglycan matrix and improving histological disc features (Patil et al., 2019). We irradiated p16-3MR mice to induce hematopoietic stem cell senescence with bone marrow suppression (Chang et al., 2016). GCV treatment could significantly decrease senescent hematopoietic stem cells, which is consistent with increasing hematopoietic stem cell engraftment by bone marrow transplantation assay. We also found that GCV treatment balanced lymphoid and myeloid cell differentiation from hematopoietic stem cells in irradiated mice. These data further confirm that hematopoietic stem cell senescence is a crucial mediator in irradiation-induced long-term bone suppression.

Overall, there are advantages and disadvantages in the five different $\mathrm{p} 16^{\mathrm{INK} 4 \mathrm{a}}$ reporter mice. We can use p16-Luc, p16 ${ }^{\mathrm{LUC}}$, and p16-3MR mice to timely monitor senescent cells because of existing luciferase in these reporters. However, it might be a challenge to isolate single $\mathrm{p} 16^{\text {INK4a }}$ positive cells due to the weak fluorochrome in INK-ATTAC and p16-3MR reporters. p16 ${ }^{\text {tdTom/t }}$ reporter has the brightest fluorochrome when compared to INK-ATTAC and p16-3MR reporters. Therefore, $\mathrm{p} 16^{\mathrm{tdTom} /+}$ reporter might be the best option to isolate and analyze single senescent cells to perform downstream analysis. To deplete senescent cells in vitro and in vivo, both INK-ATTAC and
p16-3MR mouse models can be chosen while p16-Luc, p16 ${ }^{\mathrm{LUC}}$, and $\mathrm{p} 16^{\mathrm{tdTom} /+}$ reporters cannot be used for the clearance of senescent cells.

\section{EXPRESSION OF APOPTOTIC-RELATED GENES AND SENESCENT CELL CLEARANCE}

It is well accepted that senescent cells can be monitored and depleted using five different $\mathrm{p} 16^{\mathrm{INK} 4 \mathrm{a}}$ reporter mice, mentioned above. From a therapeutic point of view, it would be better to develop applicable strategies to deplete senescent cells to counter age-related diseases. To reach that point, we must explore the molecular properties of senescent cells. Here, we mainly discussed expression of apoptotic-related genes in senescent cells, which benefits the development of mechanismbased senescent cell clearance.

\section{Expression of BCL-Family Protein in Senescent Cells}

As we mentioned above, senescent cells are more resistant to apoptosis and harsh environments, leading to their retention within tissues. Expression of $\mathrm{Bcl}-2, \mathrm{Bcl}-\mathrm{w}$, and $\mathrm{Bcl}-\mathrm{xl}$ was significantly increased in senescent IMR90 cells induced by irradiation, Ras over-expression, and replication (Yosef et al., 2016). Through ribosome sucrose gradient fractionation analysis, the increase of $\mathrm{Bcl}-\mathrm{xl}$ expression in senescent cells is shown to be due to the increased translational rates of $\mathrm{Bcl}-\mathrm{xl}$ in irradiation-induced senescent cells. Baar et al. used senescent IMR90 cells to show that expression of pro-apoptotic genes Puma and Bim were significantly upregulated (Baar et al., 2017). We also demonstrated that senescent WI-38 cells had properties with increasing expressions of pro-apoptotic Bak and anti-apoptotic Bcl-xl (Chang et al., 2016). Therefore, BCL-family proteins are an essential component of senescent cells' ability to be retained in the tissue. Expression of antiapoptotic and pro-apoptotic genes is balanced at higher levels in senescent cells compared to that in normal cells. The profile of Bcl-family gene expression suggests that senescent cells might be primed to undergo apoptosis, while the execution of the death program is temporarily suppressed (Figure 1).

Knockdown of BCL-family protein was applied to senescent cells, showing that senescent cells are sensitive to downregulation of Bcl-xl, Bcl-w, and Bcl-2 (Zhu et al., 2016). Through siRNA or shRNA techniques, the downregulation of both Bcl-xl and Bcl-w could selectively remove IMR90 and WI-38 senescent cells (Chang et al., 2016; Yosef et al., 2016). However, the downregulation of either $\mathrm{Bcl}-\mathrm{xl}$ or $\mathrm{Bcl}-\mathrm{w}$ failed to deplete senescent cells. ABT-199, a Bcl-2 inhibitor, could not clear senescent cells. Combination of ABT-199 and siRNAs against $\mathrm{Bcl}$-xl and/or Bcl-w significantly reduced the viability of senescent cells. Using pan-caspase inhibitors z-VAD-FMK or Q-VD-OPH, the depletion effects of knocking down Bcl-xl and Bcl-w on senescent cells were blocked (Chang et al., 2016; Yosef et al., 2016), indicating that the downregulation of $\mathrm{Bcl}-\mathrm{xl}$ and $\mathrm{Bcl}-\mathrm{W}$ reduces 


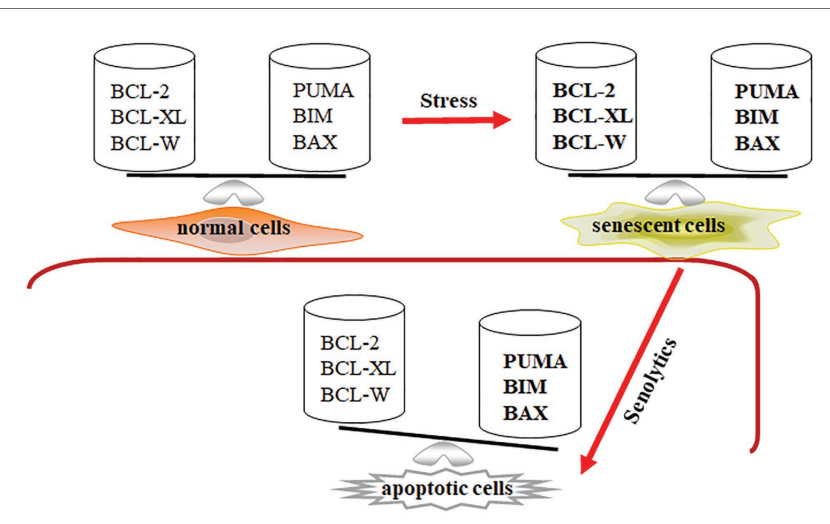

FIGURE 1 | Senescent cells are in a primed apoptotic status. In normal cells, the expression of anti-apoptotic and pro-apoptotic genes is in a wellbalanced condition. In senescent cells, the expression of anti-apoptotic and pro-apoptotic genes is abnormally upregulated (expressed in bold) and temporarily balanced, leading to primed apoptotic status for senescent cells. Decreasing expression of $\mathrm{Bcl}-2, \mathrm{Bcl}-\mathrm{xl}$, and $\mathrm{Bcl}-\mathrm{w}$ by senolytics disrupts the balance in senescent cells, leading to apoptotic cell death.

senescent cell viability via cellular apoptosis. Therefore, expression of $\mathrm{Bcl}-\mathrm{xl}, \mathrm{Bcl}-\mathrm{w}$, and $\mathrm{Bcl}-2$ contributes to the resistance of senescent cells to apoptosis. The data also suggests that inhibitors that can block Bcl-xl, Bcl-w, and Bcl-2, which may help clear senescent cells.

\section{Inhibition of Bcl-2 Family Protein Sensitizes Senescent Cells}

It has been shown that Bcl-xl, Bcl-w, and Bcl-2 inhibitors ABT-263 and ABT-737 could selectively clear senescent cells by more than $65 \%$ (Table 2), and not negatively affect normal cells (Chang et al., 2016; Yosef et al., 2016; Zhu et al., 2016). Both ABT-737 and ABT-263 treatment significantly reduced expression of $\mathrm{Bcl}-\mathrm{xl}$ and $\mathrm{Bcl}-\mathrm{w}$ in irradiation-induced lung fibrosis mouse models, which in turn decreased the numbers of senescent cells and ameliorated lung fibrosis (Pan et al., 2017). In double-transgenic K5-rtTA/tet-p14 mice, the induction of $\mathrm{p} 14^{\text {Arf }}$ expression accelerates the generation of senescent epidermal cells, which can be cleared by ABT-737 treatment (Yosef et al., 2016). Administration of ABT-737 in K5-rtTA/ tet-p14 mice benefits hair follicle stem cell proliferation, which repopulates the stem cell compartment in the bulge. We also showed that ABT-263 treatment in irradiated mice efficiently removed senescent hematopoietic and smooth muscle stem cells by increasing the stem cell's functions of self-renewal and differentiation (Chang et al., 2016). These positive effects of removing senescent stem cells on stem cells were also confirmed using p16-3MR mouse model (Chang et al., 2016). Bussian et al. used MAPT $^{\mathrm{P} 301 \mathrm{~S}}$ PS19 mouse model of tau-dependent neurodegenerative disease to investigate whether the depletion of senescent cells via ABT-263 ameliorates the tau-dependent pathology while preserving cognitive function (Bussian et al., 2018). Their data showed that administration of ABT-263 starting at weaning age prevented the upregulation of senescence-associated genes with attenuating tau phosphorylation in PS19 mice.
While studying the effects of senescent Osx $1^{+}$osteoprogenitors on bone loss with age, it was shown that ABT-263 had the ability to clear senescent Osx $1^{+}$cells with increasing osteoblasts and decreasing osteoclast formation (Kim et al., 2017). Additionally, Bcl-xl inhibitors, A1331852 and A1155463, can efficiently increase caspase $3 / 7$ activity and cellular apoptosis in senescent HUVEC and IMR90 cells (Zhu et al., 2017). The data indicates that therapeutic targeting senescent cells by senolytics can ameliorate senescent cell-mediated pathological injury. This may also suggest that senescent cells negatively affect chronic and age-related diseases.

Because ABT-263 has platelet toxicity that limits its further application, Gonzalez-Gualda et al. recently used galactoseconjugation of ABT263 to selectively induce senescent cell apoptosis without thrombocytopenia, which enhances the cytotoxicity of cisplatin in human A549 lung cancer cells (González-Gualda et al., 2020). To reduce the thrombocytopenia induced by ABT-263, Khan et al. re-constructed ABT-263 to DT2216, targeting Bcl-xl (Khan et al., 2019). They showed that DT2216 inhibited leukemia cell growth without platelet toxicity. However, the effectiveness of DT2216 on senescent cells remains unknown. Most recently, Dr. Zhou's group used proteolysis-targeting chimera technology to develop another ABT-263 mimic, PZ15227, which could selectively remove senescent cells while improving the function of tissue stem cells in naturally aged mice (He et al., 2020b). Moreover, PZ15227 does not have an in vivo thrombocytopenia effect. Therefore, it should be further tested whether PZ15227 can improve tissue function by clearing senescent cells in different aged models, such as lung fibrosis or radiation-induced chronic bone marrow damage (He et al., 2020b).

\section{Combination of Dasatinib and Quercetin Sensitizes Senescent Cells}

To investigate different approaches of removing senescent cells, Zhu et al. used transcriptional analysis using normal and senescent cells and found the increased expression of pro-survival genes, which maintains senescent cells' resistance to apoptosis (Zhu et al., 2015). Combination of dasatinib (D) and quercetin (Q) was utilized to eliminate senescent human endothelial cells, mouse bone marrow-derived mesenchymal stem cells, and fat progenitors by reducing expression of $\mathrm{p} 21^{\mathrm{Cip} 1}$, PAI-2, and $\mathrm{Bcl}-\mathrm{xl}$ in senescent cells (Table 2). Similarly, fisetin, a quercetin analog, selectively removes senescent HUVEC cells by activating the apoptotic signaling pathway (Zhu et al., 2017). Using $\mathrm{Erccl}^{-1-}$ mice as an accelerated aging model, Zhu et al. treated these aged mice with $\mathrm{D}+\mathrm{Q}$ to delete senescent cells in vivo (Zhu et al., 2015). Their data demonstrate that periodic treatment with $\mathrm{D}+\mathrm{Q}$ senolytics is sufficient to reduce senescence cells along with decreasing frailty and extending lifespan significantly. $\mathrm{D}+\mathrm{Q}$ senolytic agents enhanced cardiac and vascular function in aging mice, reduced dysfunction caused by localized irradiation, and alleviated skeletal and neurological phenotypes in progeroid mice (Zhu et al., 2015).

In idiopathic pulmonary fibrosis (IPF), fibrogenic senescent fibroblasts were selectively killed by a senolytic cocktail, D + Q (Schafer et al., 2017). It was demonstrated that early ablation 
TABLE 2 | Clearance of senescent cells by various senolytics.

\begin{tabular}{|c|c|c|c|}
\hline Senolytic agents & Senolytic targets & Killing senescent cells & Refs \\
\hline Dasatinib + Quercetin & EFNB, PI3K, P21 ${ }^{\text {Cip } 1}$, PAI2, Bcl-xI & Pre-adipocytes, HUVECs, MEFs, BM-MSCs & Zhu et al., 2015 \\
\hline ABT-263 & $\mathrm{Bcl}-2, \mathrm{Bcl}-\mathrm{xl}, \mathrm{Bcl}-\mathrm{w}$ & WI-38, IMR-90, HRECs, MEFs, HUVECs, HSCs, MuSCs & Chang et al., 2016; Zhu et al., 2016 \\
\hline ABT-737 & $\mathrm{Bcl}-2, \mathrm{Bcl}-\mathrm{xl}, \mathrm{Bcl}-\mathrm{w}$ & IMR-90, MEFs & Yosef et al., 2016 \\
\hline UBX0101 & MDM2-P53 & Chondrocytes & Jeon et al., 2017 \\
\hline FOXO4-DRI & FOXO4-P53 & IMR-90, WI-38, BJ & Baar et al., 2017 \\
\hline 17-DMAG & HSP90-AKT & MEFs, MSCs, IMR-90, WI-38 & Fuhrmann-Stroissnigg et al., 2017 \\
\hline EF24 & Bcl-xl, Mcl-1 & WI-38, IMR-90, HUVECs, HRECs, pre-adipocytes & Li et al., 2019 \\
\hline Piperlongumine (PL) & Oxidation resistance 1 & WI-38 & Wang et al., 2016; Zhang et al., 2018 \\
\hline Fisetin & $\mathrm{PI} 3 \mathrm{~K} / \mathrm{AKT}$ & HUVECs & Zhu et al., 2017 \\
\hline A1331852, A1155463 & $\mathrm{Bcl-xl}$ & HUVECs, IMR-90 & Zhu et al., 2017 \\
\hline 2-deoxy-D-glucose (2-DG) & Glycolysis & CISCs(lymphomas), vascular smooth muscle cells & Dörr et al., 2013; Gardner et al., 2015 \\
\hline Panobinostat (pano) & Histone 3 acetylation, $\mathrm{Bcl}-\mathrm{xl}$ & CISCs (NSCLC, HNSCC) & Samaraweera et al., 2017 \\
\hline PZ15227 & Bcl-xl, E3 ligase cereblon & WI-38, HREC, pre-adipocytes & He et al., 2020b \\
\hline
\end{tabular}

EFNB, ephrin ligand (EFN) B1 and B3; PI3K, phosphatidylinositol-4, 5-bisphosphate 3-kinase; PAl-2, plasminogen-activated inhibitor-2; HSP90, heat shock protein 90; HUVECs, human umbilical vein epithelial cells; MEFs, murine embryonic fibroblasts; BM-MSCs, bone marrow-derived murine mesenchymal stem cells; HRECs, human renal epithelial cells; WI-38, human lung fibroblasts; IMR-90, human lung fibroblasts; HSCs, hematopoietic stem cells; MuSCs, muscle stem cells; BJ, human foreskin fibroblasts; MSCs, murine mesenchymal stem cells; CISCs, chemotherapy-induced senescent cells; NSCLC, non-small cell lung cancer; HNSCC, head and neck squamous cell carcinoma.

of senescent cells improved pulmonary function and physical health in the bleomycin-injury IPF model, although lung fibrosis is visibly unaltered. Using $\mathrm{D}+\mathrm{Q}$ treatment, chronic clearance of senescent cells improves established vascular phenotypes associated with aging and chronic hypercholesterolemia (Roos et al., 2016). Removing senescent cells may be a viable therapeutic intervention to reduce morbidity and mortality from cardiovascular diseases (Roos et al., 2016). D + Q treatment resulted in significant reduction of senescent cells in the medial layer of aorta from aged and hypercholesterolemic mice, but not in intimal atherosclerotic plaques (Roos et al., 2016). Collectively, this study shows that chronic pharmacological clearance of senescent cells alleviates vasomotor dysfunction in naturally aged mice as well as in mice with atherosclerosis. Furthermore, senescent cell clearance reduces osteogenesis in advanced intimal plaques, ultimately reducing intimal plaque calcification. Therefore, the combination of $D+Q$ attenuates the negative effects of senescent cells in aged and progeroid mice.

\section{Regulating p53 Levels and Senescent Cell Clearance}

Irradiation- and oncogene-induced senescence are attributed to DNA damage and subsequently increase p53 expression and its activity (Sherr, 2006; Cheng and Chen, 2010; Purvis et al., 2012) (Figure 2). p53 activation not only increases pro-apoptotic gene expression with cellular apoptosis, but also leads to p21 ${ }^{\text {Cip1 }}$ upregulation and initiates cellular senescence (Karimian et al., 2016; Hafner et al., 2019). p21 $1^{\text {Cip1 }}$ overexpression promotes cell cycle arrest along with consecutive p16 ${ }^{\text {INK4a }}$ upregulation. However, p53 levels are much lower in senescent cells than those in normal cells (Kim et al., 2015; Johmura and Nakanishi, 2016), indicating that p53 activation is dispensable in senescent cells. To investigate how p53 expression was regulated in senescent cells, Sisoula et al. showed that chaperone-associated ligase CHIP was highly expressed in senescent cells, promoting p53 degradation below the basal level (Sisoula et al., 2011). Accordingly, p53 degradation was observed by the inhibition

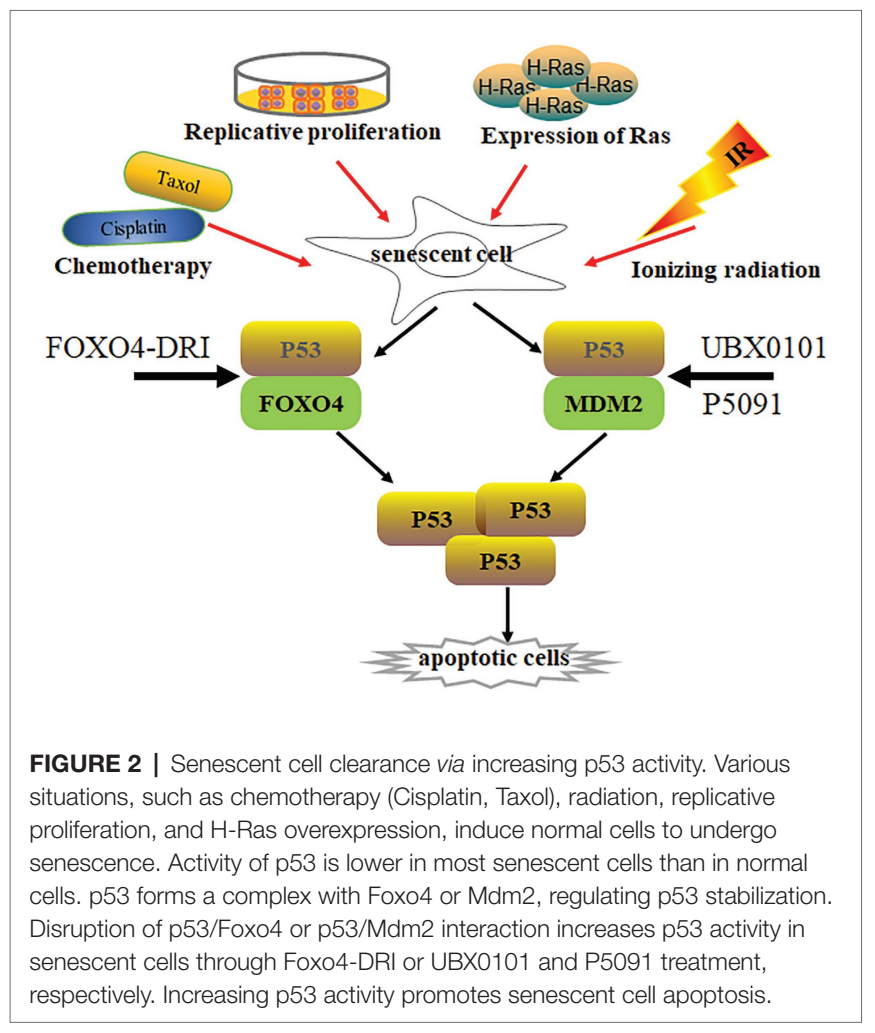

of HSP90 activity in senescent cells but not in normal cells (Sisoula et al., 2011). Johmura et al. demonstrated that SCF (Fbxo22) was highly expressed in senescent cells and formed a complex with a lysine demethylase KDM4A, which can target methylated p53, regulating p53 degradation in senescent cells (Johmura et al., 2016).

p53 is a well-known tumor suppressor that modulates cellular senescence, cancer, and aging (Hasty and Christy, 2013; Rufini et al., 2013). Is there any special functional meaning of low p53 levels in senescent cells? Whether low p53 expression accelerates senescent cell transformation remains 
elusive. On the other hand, could p53 re-activation in senescent cells resume cellular apoptotic program by increasing pro-apoptotic gene expression? An opening question for this point is how to re-activate p53 in senescent cells. Recently, Baar et al. found that Foxo4 expression was increased in senescent cells, which might promote senescent cell survival by interacting with $\mathrm{p} 53$. The interaction inhibits $\mathrm{p} 53$-mediated cellular apoptosis (Baar et al., 2017). The peptide Foxo4-DRI can promote nuclear exclusion of active pSer15-p53 and decrease p $21^{\text {Cipl }}$ expression, activating downstream target gene expression and inducing cellular-intrinsic apoptosis by caspase 3 cleavage. This resulted in clearing senescent IMR90 cells by activating the intrinsic apoptotic pathway without affecting normal cells. Foxo4-DRI also reduces in vivo doxorubicin-induced senescence and liver toxicity (Baar et al., 2017). The beneficial effects of Foxo4-DRI were also observed in fast-aging $\mathrm{Xpd}^{\mathrm{TT} / \mathrm{TT}}$ and naturally aged mice (Baar et al., 2017). The Foxo4-DRI treatment improved fur density, renal function, and physical activity in aged mice. These data prove that increasing p53 activity can selectively remove senescent cells. However, Foxo4-DRI has a short half-life in vivo (Baar et al., 2017). Thus, different strategies to increase p53 activity must be investigated to remove senescent cells.

To activate intracellular p53, Jeon utilized UBX0101, a senolytic MDM2/p53 interaction inhibitor, to increase p53 activity and stability through blocking MDM2-mediated p53 ubiquitination and proteasome degradation (Jeon et al., 2017). UBX0101 can remove senescent cells in vitro and in vivo, resulting in alleviating post-traumatic osteoarthritis, reducing pain, and increasing cartilage development. Therefore, blocking the interaction between MDM2 and p53 can prevent p53 degradation, which increases p53 activity and deplete senescent cells. Most recently, $\mathrm{He}$ et al. demonstrated that ubiquitinspecific peptidase 7 (USP7) inhibitor P5091 accelerated MDM2 ubiquitination and degradation, which in turn increased intracellular p53 expression and cleared senescent cells by activating cellular apoptosis (He et al., 2020a). The depletion of senescent cells by P5091 alleviated doxorubicin-induced SASP and renal toxicities in mice. Therefore, increasing p53 activity in senescent cells is a novel senolytic strategy, which can be achieved by disrupting MDM2/P53 interaction to regulate the stability of p53 and MDM2.

\section{Other Pro-apoptotic Strategies Sensitize Senescent Cells}

Since D + Q and ABT-263 can remove senescent cells, many other senolytics have been developed to selectively clear senescent cells. It has been reported that heat shock protein 90 (HSP90) inhibitor 17-DMAG (alvespimycin) eliminated senescent $\mathrm{Ercc1}^{-1-}$ MEFs through inducing apoptosis by inhibiting HSP90 and downregulating the anti-apoptotic PI3K/AKT pathway in vitro (Table 2). 17-DMAG treatment delays the onset of several age-related symptoms, and extends lifespan in $\mathrm{Erccl}^{-/-}$mice (Fuhrmann-Stroissnigg et al., 2017). EF24, a curcumin analog, selectively depletes senescent cells via cellular apoptotic mechanisms (Li et al., 2019). The killing effects of EF24 on senescent cells are dependent on the decreased expression of
$\mathrm{Bcl}-\mathrm{xl}$ and Mcl-1 via proteasome degradation, which induces cellular apoptosis. However, whether EF24 can remove senescent cells in vivo remains to be determined. Piperlongumine (PL) isolated from genus piper preferentially clears senescent human WI-38 fibroblasts through inducing cellular apoptosis but not ROS production (Wang et al., 2016). PL can directly bind to oxidation resistance 1 (OXR1), an important antioxidant protein that regulates the expression of a variety of antioxidant enzymes. PL selectively induces OXR1 degradation through the ubiquitin-proteasome system in a senescent cell-specific manner (Zhang et al., 2018).

2-deoxy-D-glucose (2-DG), an inhibitor of glycolysis, eliminates therapy-induced senescent cells in vivo and in vitro through caspase-12- and caspase-3-mediated endoplasmic reticulum-related apoptosis (Dörr et al., 2013). Furthermore, 2-DG leads to significant death of senescent vascular smooth muscle cells in vitro through apoptosis (Gardner et al., 2015). Panobinostat is a histone deacetylase inhibitor, which can significantly clear chemotherapy-induced senescent cells by increasing caspase 3/7 activity, decreasing expression of Bcl-xl, and increasing $\mathrm{H} 3$ acetylation in non-small cell lung cancer and head and neck squamous cell carcinoma cell lines (Samaraweera et al., 2017). Taken together, the killing mechanisms of current available senolytics are mainly through cellular apoptosis. However, these senolytics can remove around 65\% of senescent cells through activating the intrinsic apoptotic pathway. These data suggest that other mechanism-based killing approaches of senescent cells might exist and be developed in the future. Thus far, it has not been extensively investigated whether expression of extrinsic apoptotic signaling-related proteins is changed in senescent cells. Does activation of the extrinsic apoptotic signaling pathway have the ability to clear senescent cells? Activation of both intrinsic and extrinsic apoptotic signaling pathways might efficiently remove senescent cells. These questions should be further investigated.

\section{TARGETING MITOCHONDRIA AND CLEARANCE OF SENESCENT CELLS}

As we reviewed above, both ABT-737 and ABT-263 have senolytic functions on senescent cells, which is mediated by the activation of the intrinsic mitochondrial-related apoptotic pathway. However, senescent cells have robust metabolic activity with increasing oxygen consumption, mitochondrial potential, energy, and ROS production. Many studies have shown the abnormal changes of mitochondria on senescent cells. Accumulation of mitochondrial DNA (mtDNA) mutation has been implicated in age-related diseases (Hahn and Zuryn, 2019). The phenomenon is supported in mitochondrial DNA polymerase gamma (PolG) mutant mice, showing that PolG mutant mice had premature aging phenotype with mtDNA mutation accumulation (Graziewicz et al., 2007). mtDNA mutation accumulation can drive electron transport chain (ETC) dysfunction and ROS production in senescent cells, forming a "vicious cycle" and enhancing mitochondrial damage (Hahn and Zuryn, 2019). Pharmacologic inhibition 
of ETC complexes has been reported to induce oxidative stress and premature senescence in cells (Stockl et al., 2006).

Mitophagy is essential for the clearance of damaged or dysfunctional mitochondria and prevention of cellular senescence. Mitophagy is regulated by mitochondrial fission and fusion. Impaired mitochondrial fission and fusion processes negatively affect cells' ability to degrade dysfunctional mitochondria, causing ROS-induced DNA damage and senescence. Decreased Pink1 expression has detrimental effects on mitophagy and reduces the mitochondrial membrane potential along with mitochondrial dysfunction (Tsujimoto et al., 2020). Increasing mitophagy by activation of Pink1/Parkin pathway significantly decreases numbers of SA- $\beta$-gal positive cells in bronchial epithelial cells and mesenchymal stem cells (Tsujimoto et al., 2020). In addition, mitochondrial unfolded protein response (mtUPR) is initiated by cleavage of unfolded mitochondrial proteins into peptides by the protease CLPP-1. Those peptides are exported into the cytoplasm by the transporter HAF-1 (Shpilka and Haynes, 2018). Previous studies showed that dysfunction of mtUPR may play an important role in age-related pathology, which is supported by the extending lifespan under mtUPR activation conditions (Shpilka and Haynes, 2018). It is not clear whether activation of mtUPR is either sufficient for lifespan extension or necessary for lifespan extension in conditions where mitochondrial function is modulated, including caloric restriction. Other data also support that mtUPR has been shown to be necessary for enhanced survival in response to various stresses (Mao et al., 2016).

Because senescent cells have dysfunctional mitochondria with high metabolical activity, is it possible to specifically target mitochondria to remove senescent cells? Most recently, Neuzil's group investigated the role of the chemical mitochondria-targeted tamoxifen (MitoTam) on depleting senescent cells (Hubackova et al., 2019a,b). MitoTam can specifically target respiratory complex I in the mitochondrial inner membrane by interfering with electron transfer, leading to ROS production. MitoTam treatment selectively removed senescent cells from cancer and normal tissues/cells, including human RPE-1, lung fibroblast HFP-1 and foreskin fibroblast BJ cells. Moreover, MitoTam selectively kills senescent cells both in vitro and in vivo through suppressing OXPHOS and decreasing mitochondrial potential independent of increasing ROS production. The clearance efficiency of MitoTam on senescent cells is four times greater than that of ABT-737. However, other complex I inhibitors, like IACS-010759 and metformin, failed to clear senescent cells (Hubackova et al., 2019a). This data indicates that targeting respiratory complex I is not the only mechanism of MtioTam on removing senescent cells.

Further data found that senescent cells expressed lower levels of adenine nucleotide translocase-2 (ANT2) when compared to normal cells. ANT2, as a mitochondrial carrier family protein, transfers ATP generated by glycolysis into mitochondria (Fiore et al., 1998). MitoTam selectively depletes senescent cells because of downregulation of ANT2. The sensitivity of senescent cells to MitoTam treatment can be counteracted by upregulation of ANT2. Consistently, the combination of complex I inhibitor rotenone and mitochondrial uncoupler CCCP selectively causes senescent cell death (Hubackova et al., 2019a). Simultaneous interference with mitochondrial structure and integrity, and ATP hemostasis in senescent cells, leads to their effective removal. Therefore, the interaction between ANT2 and ATP synthase plays a critical role in the maintenance of mitochondrial integrity. Targeting dysfunctional mitochondria by MitoTam opens a novel avenue to remove senescent cells in senescentrelated diseases and pathologies.

\section{DISCUSSION}

We have discussed five different $\mathrm{p} 16^{\mathrm{INK} 4 \mathrm{a}}$ reporters that were used to investigate senescent cells. However, some senescent cells do not highly express p16 ${ }^{\mathrm{INK} 4 \mathrm{a}}$, and $\mathrm{p} 21^{\mathrm{Cip} 1}$ upregulation was observed in senescent cells (Herbig et al., 2004). Therefore, p $16^{\text {INK4a }}$ reporters might underestimate the actual numbers of senescent cells. It would be helpful if we could find other reporters to compensate $\mathrm{p} 16^{\text {INK4a }}$ reporters to accurately assess senescent cells. For senescent cell clearance, INK-ATTAC or p16-3MR reporters only remove senescent cells with $\mathrm{p} 16^{\mathrm{INK} 4 \mathrm{a}}$ upregulation and fail to deplete those senescent cells with low levels of $\mathrm{p} 16^{\text {INK4a }}$ expression. Therefore, INK-ATTAC or p16-3MR reporters are not sufficient to clear all senescent cells in progeroid mice. Other reporters for senescent cells should be developed to better eliminate senescent cells. It is well known that $\mathrm{p} 21^{\mathrm{Cip} 1}$ expression is increased in some senescent cells. Therefore, a p21 $1^{\text {Cipl }}$ reporter mouse might be a good option to label or remove senescent cells. A combination of $\mathrm{p} 21^{\mathrm{Cip} 1}$ reporter and INK-ATTAC or p16-3MR reporters may detect all senescent cells. This hypothesis needs to be tested in the near future.

Several senolytics have been developed to target senescent cells, but many of them have cell type specific killing effects. For example, senescent human pre-adipocytes are not sensitive to ABT-263 (Zhu et al., 2016), although ABT-263 kills more than $60 \%$ of senescent WI-38, IMR-90, HUVECs, and MEFs in $72 \mathrm{~h}$ (Chang et al., 2016). Fisetin could not eliminate senescent IMR-90 and preadipocytes, but efficiently cleared senescent HUVECs. A1331852 and A1155463 are senolytics in HUVECs and IMR-90 cells but not primary human preadipocytes (Zhu et al., 2017). Thus, it would be possible to target a specific type of senescent cells by senolytic designing. Another possible method is to combine different senolytics to clear more senescent cells. These approaches should be tested in further studies. As we discussed above, around 15\% of cells with senescent features exist in aged tissues. The function of those senescent cells might contribute to the maintenance of tissue integrity when tissues are damaged. This opens the question of whether we should remove all senescent cells or part of them to keep tissue integrity, which should be investigated in the near future as well.

It is well-known that organelle homeostasis is crucial to maintain normal cellular function. Senescent cells have multiple functional defects in mitochondria, such as impaired mitochondrial morphology, mtDNA mutation, mitophagy, and mtUPR. Through targeting mitochondria, MitoTam can efficiently remove senescent cells with low levels of ANT2 protein expression. Along with 
dysfunctional mitochondria, other cellular organelles have abnormal functions in senescent cells, such as an unfolded protein response in the endoplasmic reticulum, an increase in senescence-associated- $\beta$-galactosidase activity in lysosome, senescence-associated heterochromatin foci, and DNA damage response in nucleus (Hwang et al., 2009). To efficiently deplete senescent cells, these dysfunctional organelles might become perfect candidates to ameliorate aging-related diseases in the future.

\section{AUTHOR CONTRIBUTIONS}

YF and LS designed the study. YF, JC, HZ, and LS drafted the manuscript. All authors contributed to the article and approved the submitted version.

\section{REFERENCES}

Adams, P. D. (2009). Healing and hurting: molecular mechanisms, functions, and pathologies of cellular senescence. Mol. Cell 36, 2-14. doi: 10.1016/j. molcel.2009.09.021

Aguayo-Mazzucato, C., Andle, J., Lee, T. B. Jr., Midha, A., Talemal, L., Chipashvili, V., et al. (2019). Acceleration of beta cell aging determines diabetes and senolysis improves disease outcomes. Cell Metab. 30, 129-142.e1-e4. doi: 10.1016/j. cmet.2019.05.006

Baar, M. P., Brandt, R. M. C., Putavet, D. A., Klein, J. D. D., Derks, K. W. J., Bourgeois, B. R. M., et al. (2017). Targeted apoptosis of senescent cells restores tissue homeostasis in response to chemotoxicity and aging. Cell 169, 132-147. doi: 10.1016/j.cell.2017.02.031

Baker, D. J., Alimirah, F., van Deursen, J. M., Campisi, J., and Hildesheim, J. (2017). Oncogenic senescence: a multi-functional perspective. Oncotarget 8, 27661-27672. doi: 10.18632/oncotarget.15742

Baker, D. J., Childs, B. G., Durik, M., Wijers, M. E., Sieben, C. J., Zhong, J., et al. (2016). Naturally occurring p16 (Ink4a)-positive cells shorten healthy lifespan. Nature 530, 184-189. doi: 10.1038/nature16932

Baker, D. J., Perez-Terzic, C., Jin, F., Pitel, K. S., Niederlander, N. J., Jeganathan, K., et al. (2008). Opposing roles for p16Ink4a and p19Arf in senescence and ageing caused by BubR1 insufficiency. Nat. Cell Biol. 10, 825-836. doi: $10.1038 / \mathrm{ncb} 1744$

Baker, D. J., Wijshake, T., Tchkonia, T., LeBrasseur, N. K., Childs, B. G., Sluis, B., et al. (2011). Clearance of p16Ink4a-positive senescent cells delays ageingassociated disorders. Nature 479, 232-236. doi: 10.1038/nature10600

Barnes, P. J., Baker, J., and Donnelly, L. E. (2019). Cellular senescence as a mechanism and target in chronic lung diseases. Am. J. Respir. Crit. Care Med. 200, 556-564. doi: 10.1164/rccm.201810-1975TR

Burd, C. E., Sorrentino, J. A., Clark, K. S., Darr, D. B., Krishnamurthy, J., Deal, A. M., et al. (2013). Monitoring tumorigenesis and senescence in vivo with a p16INK4a-luciferase model. Cell 152, 340-351. doi: 10.1016/j. cell.2012.12.010

Burton, D. G. A., and Stolzing, A. (2018). Cellular senescence: immunosurveillance and future immunotherapy. Ageing Res. Rev. 43, 17-25. doi: 10.1016/j. arr.2018.02.001

Bussian, T. J., Aziz, A., Meyer, C. F., Swenson, B. L., van Deursen, J. M., and Baker, D. J. (2018). Clearance of senescent glial cells prevents tau-dependent pathology and cognitive decline. Nature 562, 578-582. doi: 10.1038/s41586018-0543-y

Calcinotto, A., Kohli, J., Zagato, E., Pellegrini, L., Demaria, M., and Alimonti, A. (2019). Cellular senescence: aging, cancer, and injury. Physiol. Rev. 99, 1047-1078. doi: 10.1152/physrev.00020.2018

Chang, J., Wang, Y., Shao, L., Laberge, R. M., Demaria, M., Campisi, J., et al. (2016). Clearance of senescent cells by ABT263 rejuvenates aged hematopoietic stem cells in mice. Nat. Med. 22, 78-83. doi: 10.1038/nm.4010

Cheng, Q., and Chen, J. (2010). Mechanism of p53 stabilization by ATM after DNA damage. Cell Cycle 9, 472-478. doi: 10.4161/cc.9.3.10556

\section{FUNDING}

The study was supported in part by the National Natural Science Foundation of China (grant no. 81860026, 81960104, and 82073484), Key R\&D plan of Jiangxi Provincial Science and Technology Department (grant no. 20192BBG70043), and the Graduate Innovation Special Fund of Jiangxi Province (grant no. CX2019126 and CX2019124).

\section{ACKNOWLEDGMENTS}

Our thanks go to Professor Qingxian Zhu (Nanchang University, China), who provided helpful suggestions on the discussion section of p16INK4a reporter mouse models.

Childs, B. G., Baker, D. J., Wijshake, T., Conover, C. A., Campisi, J., and Deursen, J. M. (2016). Senescent intimal foam cells are deleterious at all stages of atherosclerosis. Science 354, 472-477. doi: 10.1126/science.aaf6659

Childs, B. G., Durik, M., Baker, D. J., and van Deursen, J. M. (2015). Cellular senescence in aging and age-related disease: from mechanisms to therapy. Nat. Med. 21, 1424-1435. doi: 10.1038/nm.4000

Childs, B. G., Gluscevic, M., Baker, D. J., Laberge, R. M., Marquess, D., Dananberg, J., et al. (2017). Senescent cells: an emerging target for diseases of ageing. Nat. Rev. Drug Discov. 16, 718-735. doi: 10.1038/ nrd.2017.116

Collado, M., Blasco, M. A., and Serrano, M. (2007). Cellular senescence in cancer and aging. Cell 130, 223-233. doi: 10.1016/j.cell.2007.07.003

Coppé, J. -P., Desprez, P. -Y., Krtolica, A., and Campisi, J. (2010). The senescenceassociated secretory phenotype: the dark side of tumor suppression. Annu. Rev. Pathol. 5, 99-118. doi: 10.1146/annurev-pathol-121808-102144

Coppé, J. -P., Patil, C. K., Rodier, F., Sun, Y., Munoz, D. P., Goldstein, J., et al. (2008). Senescence-associated secretory phenotypes reveal cell-nonautonomous functions of oncogenic RAS and the p53 tumor suppressor. PLoS Biol. 6, 2853-2868. doi: 10.1371/journal.pbio.0060301

d'Adda di Fagagna, F. (2008). Living on a break: cellular senescence as a DNAdamage response. Nat. Rev. Cancer 8, 512-522. doi: 10.1038/nrc2440

Davaapil, H., Brockes, J. P., and Yun, M. H. (2017). Conserved and novel functions of programmed cellular senescence during vertebrate development. Development 144, 106-114. doi: 10.1242/dev.138222

Demaria, M., Ohtani, N., Youssef, S. A., Rodier, F., Toussaint, W., Mitchell, J. R., et al. (2014). An essential role for senescent cells in optimal wound healing through secretion of PDGF-AA. Dev. Cell 31, 722-733. doi: 10.1016/j. devcel.2014.11.012

Dimri, G. P., Lee, X., Basile, G., Acosta, M., Scott, G., Roskelley, C., et al. (1995). A biomarker that identifies senescent human cells in culture and in aging skin in vivo. Proc. Natl. Acad. Sci. U. S. A. 92, 9363-9367. doi: 10.1073/pnas.92.20.9363

Docherty, M. H., O'Sullivan, E. D., Bonventre, J. V., and Ferenbach, D. A. (2019). Cellular senescence in the kidney. J. Am. Soc. Nephrol. 30, 726-736. doi: 10.1681/ASN.2018121251

Dörr, J. R., Yu, Y., Milanovic, M., Beuster, G., Zasada, C., Däbritz, J. M., et al. (2013). Synthetic lethal metabolic targeting of cellular senescence in cancer therapy. Nature 501, 421-425. doi: 10.1038/nature12437

Farr, J. N., Xu, M., Weivoda, M. M., Monroe, D. G., Fraser, D. G., Onken, J. L., et al. (2017). Targeting cellular senescence prevents age-related bone loss in mice. Nat. Med. 23, 1072-1079. doi: 10.1038/nm.4385

Fiore, C., Trézéguet, V., Le Saux, A., Roux, P., Schwimmer, C., Dianoux, A. C., et al. (1998). The mitochondrial ADP/ATP carrier: structural, physiological and pathological aspects. Biochimie 80, 137-150. doi: 10.1016/S0300 9084(98)80020-5

Fuhrmann-Stroissnigg, H., Ling, Y. Y., Zhao, J., McGowan, S. J., Zhu, Y., Brooks, R. W., et al. (2017). Identification of HSP90 inhibitors as a novel class of senolytics. Nat. Commun. 8:422. doi: 10.1038/s41467-017-00314-z 
Gadd, M., Pisc, C., Branda, J., Ionescu-Tiba, V., Nikolic, Z., Yang, C., et al. (2001). Regulation of cyclin D1 and p16(INK4A) is critical for growth arrest during mammary involution. Cancer Res. 61, 8811-8819.

Gardner, S. E., Humphry, M., Bennett, M. R., and Clarke, M. C. H. (2015). Senescent vascular smooth muscle cells drive inflammation through an interleukin-1 $\alpha$-dependent senescence-associated secretory phenotype. Arterioscler. Thromb. Vasc. Biol. 35, 1963-1974. doi: 10.1161/ATVBAHA.115.305896

Gil, J., and Peters, G. (2006). Regulation of the INK4b-ARF-INK4a tumour suppressor locus: all for one or one for all. Nat. Rev. Mol. Cell Biol. 7, 667-677. doi: $10.1038 / \mathrm{nrm} 1987$

González-Gualda, E., Pàez-Ribes, M., Lozano-Torres, B., Macias, D., Wilson, J. R., González-López, C., et al. (2020). Galacto-conjugation of Navitoclax as an efficient strategy to increase senolytic specificity and reduce platelet toxicity. Aging Cell 19:e13142. doi: 10.1111/acel.13142

Gorgoulis, V., Adams, P. D., Alimonti, A., Bennett, D. C., Bischof, O., Bishop, C., et al. (2019). Cellular senescence: defining a path forward. Cell 179, 813-827. doi: $10.1016 /$ j.cell.2019.10.005

Graziewicz, M. A., Bienstock, R. J., and Copeland, W. C. (2007). The DNA polymerase gamma Y955C disease variant associated with $\mathrm{PEO}$ and parkinsonism mediates the incorporation and translesion synthesis opposite 7,8-dihydro-8-oxo-2'-deoxyguanosine. Hum. Mol. Genet. 16, 2729-2739. doi: $10.1093 / \mathrm{hmg} / \mathrm{ddm} 227$

Hafner, A., Bulyk, M. L., Jambhekar, A., and Lahav, G. (2019). The multiple mechanisms that regulate p53 activity and cell fate. Nat. Rev. Mol. Cell Biol. 20, 199-210. doi: 10.1038/s41580-019-0110-x

Hahn, A., and Zuryn, S. (2019). Mitochondrial genome (mtDNA) mutations that generate reactive oxygen species. Antioxidants 8:392. doi: 10.3390/ antiox8090392

Hara, E., Smith, R., Parry, D., Tahara, H., Stone, S., and Peters, G. (1996). Regulation of p16CDKN2 expression and its implications for cell immortalization and senescence. Mol. Cell. Biol. 16, 859-867. doi: 10.1128/ MCB.16.3.859

Hasty, P., and Christy, B. A. (2013). p53 as an intervention target for cancer and aging. Pathobiol. Aging Age Relat. Dis. 3:22702. doi: 10.3402/pba.v3i0.22702

Hayflick, L. (1965). The limited in vitro lifespan of human diploid cell strains. Exp. Cell Res. 37, 614-636. doi: 10.1016/0014-4827(65)90211-9

Hayflick, L., and Moorhead, P. S. (1961). The serial cultivation of human diploid cell strains. Exp. Cell Res. 25, 585-621. doi: 10.1016/00144827(61)90192-6

He, Y., Li, W., Lv, D., Zhang, X., Zhang, X., Ortiz, Y. T., et al. (2020a). Inhibition of USP7 activity selectively eliminates senescent cells in part via restoration of p53 activity. Aging Cell 19:e13117. doi: 10.1111/acel.13117

He, S., and Sharpless, N. E. (2017). Senescence in health and disease. Cell 169, 1000-1011. doi: 10.1016/j.cell.2017.05.015

He, Y., Zhang, X., Chang, J., Kim, H. N., Zhang, P., Wang, Y., et al. (2020b). Using proteolysis-targeting chimera technology to reduce navitoclax platelet toxicity and improve its senolytic activity. Nat. Commun. 11:1996. doi: 10.1038/s41467-020-15838-0

Herbig, U., Ferreira, M., and Condel, L. (2006). Cellular senescence in aging primates. Science 311:1257. doi: 10.1126/science.1122446

Herbig, U., Jobling, W. A., Chen, B. P. C., Chen, D. J., and Sedivy, J. M. (2004). Telomere shortening triggers senescence of human cells through a pathway involving ATM, p53, and p21(CIP1), but not p16(INK4a). Mol. Cell 14, 501-513. doi: 10.1016/S1097-2765(04)00256-4

Hernandez-Segura, A., Nehme, J., and Demaria, M. (2018). Hallmarks of cellular senescence. Trends Cell Biol. 28, 436-453. doi: 10.1016/j.tcb.2018.02.001

Herranz, N., and Gil, J. (2018). Mechanisms and functions of cellular senescence. J. Clin. Invest. 128, 1238-1246. doi: 10.1172/JCI95148

Hewitt, G., Jurk, D., Marques, F. D. M., Correia-Melo, C., Hardy, T., Gackowska, A., et al. (2012). Telomeres are favoured targets of a persistent DNA damage response in ageing and stress-induced senescence. Nat. Commun. 3:708. doi: 10.1038/ncomms1708

Hubackova, S., Davidova, E., Rohlenova, K., Stursa, J., Werner, L., Andera, L., et al. (2019a). Selective elimination of senescent cells by mitochondrial targeting is regulated by ANT2. Cell Death Differ. 26, 276-290. doi: 10.1038/ s41418-018-0118-3

Hubackova, S., Magalhaes Novais, S., Davidova, E., Neuzil, J., and Rohlena, J. (2019b). Mitochondria-driven elimination of cancer and senescent cells. Biol. Chem. 400, 141-148. doi: 10.1515/hsz-2018-0256
Hwang, E. S., Yoon, G., and Kang, H. T. (2009). A comparative analysis of the cell biology of senescence and aging. Cell. Mol. Life Sci. 66, 2503-2524. doi: 10.1007/s00018-009-0034-2

Jeon, O. H., Kim, C., Laberge, R. M., Demaria, M., Rathod, S., Vasserot, A. P., et al. (2017). Local clearance of senescent cells attenuates the development of post-traumatic osteoarthritis and creates a pro-regenerative environment. Nat. Med. 23, 775-781. doi: 10.1038/nm.4324

Johmura, Y., and Nakanishi, M. (2016). Multiple facets of p53 in senescence induction and maintenance. Cancer Sci. 107, 1550-1555. doi: 10.1111/cas.13060

Johmura, Y., Sun, J., Kitagawa, K., Nakanishi, K., Kuno, T., Naiki-Ito, A., et al. (2016). SCF (Fbxo22)-KDM4A targets methylated p53 for degradation and regulates senescence. Nat. Commun. 7:10574. doi: 10.1038/ncomms10574

Kang, T. W., Yevsa, T., Woller, N., Hoenicke, L., Wuestefeld, T., Dauch, D., et al. (2011). Senescence surveillance of pre-malignant hepatocytes limits liver cancer development. Nature 479, 547-551. doi: 10.1038/nature10599

Karimian, A., Ahmadi, Y., and Yousefi, B. (2016). Multiple functions of p21 in cell cycle, apoptosis and transcriptional regulation after DNA damage. DNA Repair 42, 63-71. doi: 10.1016/j.dnarep.2016.04.008

Khan, S., Zhang, X., Lv, D., Zhang, Q., He, Y., Zhang, P., et al. (2019). A selective BCL-XL PROTAC degrader achieves safe and potent antitumor activity. Nat. Med. 25, 1938-1947. doi: 10.1038/s41591-019-0668-Z

Kim, H. N., Chang, J., Shao, L., Han, L., Iyer, S., Manolagas, S. C., et al. (2017). DNA damage and senescence in osteoprogenitors expressing Osxl may cause their decrease with age. Aging Cell 16, 693-703. doi: 10.1111/ acel.12597

Kim, R. H., Kang, M. K., Kim, T., Yang, P., Bae, S., Williams, D. W., et al. (2015). Regulation of p53 during senescence in normal human keratinocytes. Aging Cell 14, 838-846. doi: 10.1111/acel.12364

Krishnamurthy, J., Torrice, C., Ramsey, M. R., Kovalev, G. I., Al-Regaiey, K., Su, L., et al. (2004). Ink4a/Arf expression is a biomarker of aging. J. Clin. Invest. 114, 1299-1307. doi: 10.1172/JCI22475

Kurz, D. J., Decary, S., Hong, Y., and Erusalimsky, J. D. (2000). Senescenceassociated (beta)-galactosidase reflects an increase in lysosomal mass during replicative ageing of human endothelial cells. J. Cell Sci. 113, 3613-3622. doi: 10.1023/A:1005629427630

Laberge, R. M., Adler, D., DeMaria, M., Mechtouf, N., Teachenor, R., Cardin, G. B., et al. (2013). Mitochondrial DNA damage induces apoptosis in senescent cells. Cell Death Dis. 4:e727. doi: 10.1038/cddis.2013.199

Lange, T. (2005). Shelterin: the protein complex that shapes and safeguards human telomeres. Genes Dev. 19, 2100-2110. doi: 10.1101/gad.1346005

Lewis-McDougall, F. C., Ruchaya, P. J., Domenjo-Vila, E., Shin Teoh, T., Prata, L., Cottle, B. J., et al. (2019). Aged-senescent cells contribute to impaired heart regeneration. Aging Cell 18:e12931. doi: 10.1111/acel.12931

Li, W., He, Y., Zhang, R., Zheng, G., and Zhou, D. (2019). The curcumin analog EF24 is a novel senolytic agent. Aging-Us 11, 771-782. doi: 10.18632/ aging. 101787

Liu, Y., Sanoff, H. K., Cho, H., Burd, C. E., Torrice, C., Ibrahim, J. G., et al. (2009). Expression of p16(INK4a) in peripheral blood T-cells is a biomarker of human aging. Aging Cell 8, 439-448. doi: 10.1111/j.1474-9726.2009.00489.x

Liu, J. Y., Souroullas, G. P., Diekman, B. O., Krishnamurthy, J., Hall, B. M., Sorrentino, J. A., et al. (2019). Cells exhibiting strong p16 (INK4a) promoter activation in vivo display features of senescence. Proc. Natl. Acad. Sci. U. S. A 116, 2603-2611. doi: 10.1073/pnas.1818313116

Magalhaes, J. P., and Passos, J. F. (2018). Stress, cell senescence and organismal ageing. Mech. Ageing Dev. 170, 2-9. doi: 10.1016/j.mad.2017.07.001

Mao, X. R., Kaufman, D. M., and Crowder, C. M. (2016). Nicotinamide mononucleotide adenylyltransferase promotes hypoxic survival by activating the mitochondrial unfolded protein response. Cell Death Dis. 7:e2113. doi: 10.1038/cddis. 2016.5

McHugh, D., and Gil, J. (2018). Senescence and aging: causes, consequences, and therapeutic avenues. J. Cell Biol. 217, 65-77. doi: 10.1083/jcb.201708092

Munoz-Espin, D., Canamero, M., Maraver, A., Gomez-Lopez, G., Contreras, J., Murillo-Cuesta, S., et al. (2013). Programmed cell senescence during mammalian embryonic development. Cell 155, 1104-1118. doi: 10.1016/j. cell.2013.10.019

Munoz-Espin, D., and Serrano, M. (2014). Cellular senescence: from physiology to pathology. Nat. Rev. Mol. Cell Biol. 15, 482-496. doi: 10.1038/nrm3823

Niedernhofer, L. J., Garinis, G. A., Raams, A., Lalai, A. S., Robinson, A. R., Appeldoorn, E., et al. (2006). A new progeroid syndrome reveals that 
genotoxic stress suppresses the somatotroph axis. Nature 444, 1038-1043. doi: $10.1038 /$ nature05456

Ohtani, N., Yamakoshi, K., Takahashi, A., and Hara, E. (2010). Real-time in vivo imaging of pl6gene expression: a new approach to study senescence stress signaling in living animals. Cell Div. 5:1. doi: 10.1186/1747-1028-5-1

Olovnikov, A. M. (1973). A theory of marginotomy: the incomplete copying of template margin in enzymic synthesis of polynucleotides and biological significance of the phenomenon. J. Theor. Biol. 41, 181-190. doi: 10.1016/00225193(73)90198-7

Ortega, S., Malumbres, M., and Barbacid, M. (2002). Cyclin D-dependent kinases, INK4 inhibitors and cancer. Biochim. Biophys. Acta 1602, 73-87. doi: 10.1016/s0304-419x(02)00037-9

Pan, J., Li, D., Xu, Y., Zhang, J., Wang, Y., Chen, M., et al. (2017). Inhibition of Bcl-2/xl with ABT-263 selectively kills senescent type II pneumocytes and reverses persistent pulmonary fibrosis induced by ionizing radiation in mice. Int. J. Radiat. Oncol. Biol. Phys. 99, 353-361. doi: 10.1016/j.ijrobp.2017.02.216

Patil, P., Dong, Q., Wang, D., Chang, J., Wiley, C., Demaria, M., et al. (2019). Systemic clearance of p16(INK4a) -positive senescent cells mitigates ageassociated intervertebral disc degeneration. Aging Cell 18:e12927. doi: 10.1111/ acel.12927

Prata, L., Ovsyannikova, I. G., Tchkonia, T., and Kirkland, J. L. (2018). Senescent cell clearance by the immune system: emerging therapeutic opportunities. Semin. Immunol. 40:101275. doi: 10.1016/j.smim.2019.04.003

Purvis, J. E., Karhohs, K. W., Mock, C., Batchelor, E., Loewer, A., and Lahav, G. (2012). p53 dynamics control cell fate. Science 336, 1440-1444. doi: 10.1126/ science. 1218351

Ressler, S., Bartkova, J., Niederegger, H., Bartek, J., Scharffetter-Kochanek, K., Jansen-Dürr, P., et al. (2006). pl6INK4A is a robust in vivo biomarker of cellular aging in human skin. Aging Cell 5, 379-389. doi: 10.1111/j.1474-97 26.2006.00231.x

Roos, C. M., Zhang, B., Palmer, A. K., Ogrodnik, M. B., Pirtskhalava, T., Thalji, N. M., et al. (2016). Chronic senolytic treatment alleviates established vasomotor dysfunction in aged or atherosclerotic mice. Aging Cell 15, 973-977. doi: 10.1111/acel.12458

Rufini, A., Tucci, P., Celardo, I., and Melino, G. (2013). Senescence and aging: the critical roles of p53. Oncogene 32, 5129-5143. doi: 10.1038/onc.2012.640

Sagiv, A., Biran, A., Yon, M., Simon, J., Lowe, S. W., and Krizhanovsky, V. (2013). Granule exocytosis mediates immune surveillance of senescent cells. Oncogene 32, 1971-1977. doi: 10.1038/onc.2012.206

Samaraweera, L., Adomako, A., Rodriguez-Gabin, A., and McDaid, H. M. (2017). A novel indication for panobinostat as a senolytic drug in NSCLC and HNSCC. Sci. Rep. 7:1900. doi: 10.1038/s41598-017-01964-1

Schafer, M. J., White, T. A., Iijima, K., Haak, A. J., Ligresti, G., Atkinson, E. J., et al. (2017). Cellular senescence mediates fibrotic pulmonary disease. Nat. Commun. 8:14532. doi: 10.1038/ncomms14532

Sedelnikova, O. A., Horikawa, I., Zimonjic, D. B., Popescu, N. C., Bonner, W. M., and Barrett, J. C. (2004). Senescing human cells and ageing mice accumulate DNA lesions with unrepairable double-strand breaks. Nat. Cell Biol. 6, 168-170. doi: 10.1038/ncb1095

Sherr, C. J. (2000). The Pezcoller lecture: cancer cell cycles revisited. Cancer Res. 60, 3689-3695.

Sherr, C. J. (2006). Divorcing ARF and p53: an unsettled case. Nat. Rev. Cancer 6, 663-673. doi: 10.1038/nrc1954

Shpilka, T., and Haynes, C. M. (2018). The mitochondrial UPR: mechanisms, physiological functions and implications in ageing. Nat. Rev. Mol. Cell Biol. 19, 109-120. doi: 10.1038/nrm.2017.110

Simay, Y. D., Ozdemir, A., Ibisoglu, B., and Ark, M. (2018). The connection between the cardiac glycoside-induced senescent cell morphology and rho/ rho kinase pathway. Cytoskeleton 75, 461-471. doi: 10.1002/cm.21502
Sisoula, C., Trachana, V., Patterson, C., and Gonos, E. S. (2011). CHIP-dependent p53 regulation occurs specifically during cellular senescence. Free Radic. Biol. Med. 50, 157-165. doi: 10.1016/j.freeradbiomed.2010.10.701

Stockl, P., Hutter, E., Zwerschke, W., and Jansen-Durr, P. (2006). Sustained inhibition of oxidative phosphorylation impairs cell proliferation and induces premature senescence in human fibroblasts. Exp. Gerontol. 41, 674-682. doi: 10.1016/j.exger.2006.04.009

Storer, M., Mas, A., Robert-Moreno, A., Pecoraro, M., Ortells, M. C., Di Giacomo, V., et al. (2013). Senescence is a developmental mechanism that contributes to embryonic growth and patterning. Cell 155, 1119-1130. doi: 10.1016/j. cell.2013.10.041

Tchkonia, T., and Kirkland, J. L. (2018). Aging, cell senescence, and chronic disease: emerging therapeutic strategies. JAMA 320, 1319-1320. doi: 10.1001/ jama.2018.12440

Tchkonia, T., Zhu, Y., van Deursen, J., Campisi, J., and Kirkland, J. L. (2013). Cellular senescence and the senescent secretory phenotype: therapeutic opportunities. J. Clin. Invest. 123, 966-972. doi: 10.1172/JCI64098

Tsujimoto, T., Mori, T., Houri, K., Onodera, Y., Takehara, T., Shigi, K., et al. (2020). miR-155 inhibits mitophagy through suppression of BAG5, a partner protein of PINK1. Biochem. Biophys. Res. Commun. 523, 707-712. doi: 10.1016/j.bbrc.2020.01.022

Wang, Y., Chang, J., Liu, X., Zhang, X., Zhang, S., Zhang, X., et al. (2016). Discovery of piperlongumine as a potential novel lead for the development of senolytic agents. Aging-Us 8, 2915-2926. doi: 10.18632/aging.101100

Wiley, C. D., and Campisi, J. (2016). From ancient pathways to aging cellsconnecting metabolism and cellular senescence. Cell Metab. 23, 1013-1021. doi: 10.1016/j.cmet.2016.05.010

Yamakoshi, K., Takahashi, A., Hirota, F., Nakayama, R., Ishimaru, N., Kubo, Y., et al. (2009). Real-time in vivo imaging of p16Ink4a reveals cross talk with p53. J. Cell Biol. 186, 393-407. doi: 10.1083/jcb.200904105

Yosef, R., Pilpel, N., Tokarsky-Amiel, R., Biran, A., Ovadya, Y., Cohen, S., et al. (2016). Directed elimination of senescent cells by inhibition of BCL-W and BCL-XL. Nat. Commun. 7:11190. doi: 10.1038/ncomms11190

Zhang, X., Zhang, S., Liu, X., Wang, Y., Chang, J., Zhang, X., et al. (2018). Oxidation resistance 1 is a novel senolytic target. Aging Cell 17:e12780. doi: 10.1111 /acel. 12780

Zhu, Y., Doornebal, E. J., Pirtskhalava, T., Giorgadze, N., Wentworth, M., Fuhrmann-Stroissnigg, H., et al. (2017). New agents that target senescent cells: the flavone, fisetin, and the BCL-XL inhibitors, A1331852 and A1155463. Aging-Us 9, 955-963. doi: 10.18632/aging.101202

Zhu, Y., Tchkonia, T., Fuhrmann-Stroissnigg, H., Dai, H. M., Ling, Y. Y., Stout, M. B., et al. (2016). Identification of a novel senolytic agent, navitoclax, targeting the Bcl-2 family of anti-apoptotic factors. Aging Cell 15, 428-435. doi: 10.1111/acel.12445

Zhu, Y., Tchkonia, T., Tamar Pirtskhalava, T., Gower, A. C., Ding, H., Giorgadze, N., et al. (2015). The Achilles' heel of senescent cells: from transcriptome to senolytic drugs. Aging Cell 14, 644-658. doi: 10.1111/acel.12344

Conflict of Interest: The authors declare that the research was conducted in the absence of any commercial or financial relationships that could be construed as a potential conflict of interest.

Copyright (c) 2020 Fan, Cheng, Zeng and Shao. This is an open-access article distributed under the terms of the Creative Commons Attribution License (CC BY). The use, distribution or reproduction in other forums is permitted, provided the original author(s) and the copyright owner(s) are credited and that the original publication in this journal is cited, in accordance with accepted academic practice. No use, distribution or reproduction is permitted which does not comply with these terms. 